INSTITUT NATIONAL DE RECHERCHE EN INFORMATIQUE ET EN AUTOMATIQUE

Ion flux, transmembrane potential, and osmotic stabilization: A new electrophysiological dynamic model for Eukaryotic cells

Clair Poignard — Aude Silve — Frederic Campion — Lluis M. Mir — Olivier Saut — Laurent Schwartz

$\mathbf{N}^{\circ} \mathbf{7 2 6 9}$

Avril 2010

Thème NUM

apport

de recherche 



\title{
Ion flux, transmembrane potential, and osmotic stabilization: A new electrophysiological dynamic model for Eukaryotic cells
}

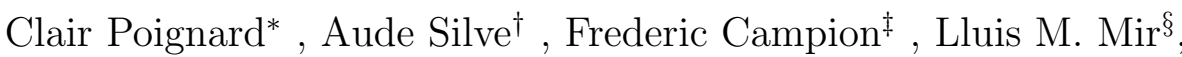 \\ Olivier Saut T], Laurent Schwartz \\ Thème NUM - Systèmes numériques \\ Équipes-Projets MC2
}

Rapport de recherche $\mathrm{n}^{\circ} 7269$ - Avril $2010-20$ pages

\begin{abstract}
Survival of mammalian cells is achieved by tight control of cell volume while transmembrane potential is known to control many cellular functions since the seminal work of Hodgkin and Huxley. Regulation of cell volume and transmembrane potential have a wide range of implications in physiology, from neurological and cardiac disorders to cancer and muscle fatigue. Therefore understanding the relationship between transmembrane potential, ion fluxes, and cell volume regulation has become of great interest. In this paper we derive a system of differential equations that links transmembrane potential, ionic concentrations, and cell volume. This model demonstrates that volume stabilization occurs within minutes of changes in extracellular osmotic pressure. We infer a straightforward relationship between transmembrane potential and cell volume. Our model is a generalization of previous models in which either cell volume was constant or osmotic regulation instantaneous. When the extracellular osmotic pressure is constant, the cell volume varies as a function of transmembrane potential and ions fluxes thus providing an implicit link between transmembrane potential and cell growth. Numerical simulations of the model provide results that are consistent with experimental data in terms of time-related changes in cell volume and dynamics of the phenomena.
\end{abstract}

Key-words: Electric potential - Cell volume regulation - Ion concentration - Membrane permeability - Osmotic equilibrium

* INRIA Bordeaux-Sud-Ouest, Institut de Mathématiques de Bordeaux, CNRS UMR 5251 \& Université de Bordeaux1, 351 cours de la Libération, 33405 Talence Cedex, France

$\dagger$ CNRS, UMR 8203, Laboratory of Vectorology and Anticancer Therapies, Institut Gustave Roussy\& Université Paris-Sud. 114, rue Edouard Vaillant, 94805 Villejuif, France.

$\ddagger$ The Copenhagen Muscle Research Centre, Rigshospitalet, 2200 Copenhagen N, Denmark.

$\S$ CNRS, UMR 8203, Laboratory of Vectorology and Anticancer Therapies, Institut Gustave Roussy\& Université Paris-Sud. 114, rue Edouard Vaillant, 94805 Villejuif, France.

ฯ INRIA Bordeaux-Sud-Ouest, Institut de Mathématiques de Bordeaux, CNRS UMR 5251

\& Université de Bordeaux1, 351 cours de la Libération, 33405 Talence Cedex, France

|| Service de Radiothérapie, Hôpital Universitaire Pitié-Salpétrière, 75013 Paris, France \& LIX, Laboratoire d'Informatique, Ecole Polytechnique

Centre de recherche INRIA Bordeaux - Sud Ouest Domaine Universitaire - 351, cours de la Libération 33405 Talence Cedex Téléphone : +33540006900 
Flux ioniques, potentiel transmembranaire et stabilisation osmotique: un modèle dynamique d'électrophysiologie pour les cellules eucaryotes

Résumé : Pas de résumé

Mots-clés : Potentiel Electrique de Membrane - Régulation du volume cellulaire - Concentration ionique - Perméabilité membranaire - Equilibre osmotique 


\section{Contents}

1 Introduction 4

\begin{tabular}{|llll}
2 & The transmembrane potential & 5
\end{tabular}

2.1 Ion flux through channels . . . . . . . . . . . . . . . . 5

2.2 Evolution of the ionic concentrations . . . . . . . . . . . . . 7

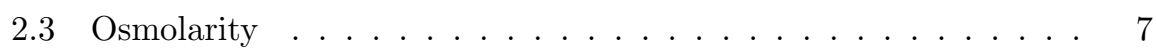

2.4 Equations for the transmembrane potential . . . . . . . . . . 8

2.5 Explicit link between the cell radius and the potential . . . . . . 9

2.5.a The case of spherical cells . . . . . . . . . . . . 10

$2.6 \quad$ Explicit formula for the transmembrane potential. . . . . . . . . 10

\begin{tabular}{|lll}
3 & Numerical simulations & 11
\end{tabular}

$3.1 \quad$ Effect of an osmotic stress on the transmembrane potential and

3.1.a Hyperosmotic stress . . . . . . . . . . . . . . . . . . . . . . . . . . . . . . . . . 13

3.1.b Hypo-osmotic stress . . . . . . . . . . . . . . . . . 13

3.1.c Influence of the coefficient $\tau_{w}$ on the dynamics . . . . . . 14

\begin{tabular}{llr}
\hline & Preliminary experiments & $\mathbf{1 5}$
\end{tabular}

4.1 Materials and Methods . . . . . . . . . . . . . . . . . . . . 15

4.1.a Cell culture . . . . . . . . . . . . . . . . 15

4.1.b Solutions used . . . . . . . . . . . . . . . . . . . . 16

4.1.c Fluorescence Activated Cell Sorter (FACS) experiments . 16

4.2 Statistical analysis of FSC measurements . . . . . . . . . . 16

4.3 Results . . . . . . . . . . . . . . . . . . 16

$\begin{array}{lll}5 & \text { Discussion } & 18\end{array}$

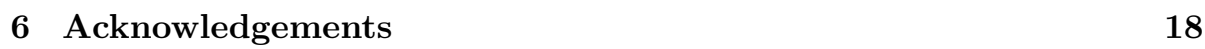




\section{Introduction}

Cell volume and resting transmembrane potential constitute two fundamental parameters of cell life. A cell contains inside its cytoplasm substances such as DNA, amino acids, ions like $\mathrm{K}^{+}$or $\mathrm{Na}^{+}$and sugar, which are essential to its survival. This synthetic process favors swelling of the cell [17, since the membrane permeability is very low. Without volume changes, the pressure created by the osmolarity difference between the outer medium and the cytoplasm could reach an atmosphere [1, 7, which is much larger than the maximal pressure that the cell membrane can withstand. Whereas plasmalemma of bacteria are protected by a thick peptidoglycan layer that can support such pressures, Eukaryotic cells have no wall and therefore must adjust their osmolarity by transferring ions and water accross their membrane. Over many years, it became clear that the osmotic stabilization of living Eukaryotic cells was related to the active transport of $\mathrm{Na}^{+}$out of and $K^{+}$into the cell [9, 16, 11, 12. These ion fluxes create a potential difference across the membrane that changes the value of the transmembrane potential. Therefore the cell volume regulation and the transmembrane potential are intrinsically correlated.

Based on the extensive review of Fraser and Huang [7, 8], all theoretical models (to our knowledge) describing the behavior of the transmembrane potential, of the ionic fluxes, and of the cell volume consider that the water flux across the membrane is instantaneous. Therefore an osmotic equilibrium of the cell is constantly required [1, 5, 7. However, such a requirement does not explain the delay in the experiments reported by Rouzaire-Dubois et al. [21, 4, 22. in the osmotic stabilization of the cell, when a high osmotic stress is imposed. Actually, when the ionic fluxes across the membrane are small, the osmotic equilibrium can be considered constant as supposed by Fraser and Huang [7, 8] or Armstrong [1] for instance. However should the cell be subjected to a large osmotic stress, then water flux across the membrane must be accounted for.

In this paper we aim to clarify the relationship between cell volume, transmembrane potential and ion fluxes. More precisely, we aim at providing a new electrophysiological model of the cell in order to dynamically link the cell volume, the ionic concentrations, and the transmembrane potential by considering the water flux accross the membrane. Our model is a generalization of the previous models in the sense that it describes the models of Jakobsson [14], Armstrong [1, Fraser and Huang [7] or Endresen et al. [5] under their respective restrictive hypotheses. Our goal is also to propose an explanation for the results presented by Rouzaire-Dubois et al. [21, 4, 22.

In order to derive our model, we consider in vitro cell cultures and more precisely spherical cells in suspension. The extracellular ionic concentrations are supposed constant and the cell volume regulation occurs homothetically (i.e. equivalently in all directions). These assumptions are justified for in vitro experiments since the volume of the ambient medium is much larger than the cell volume and since no mechanical stress is imposed to the cell. Experimentally, the osmotic equilibrium is not enforced instantaneously contrary to the assumptions of the models of Jakobsson [14], Armstrong [1], Fraser and Huang [7, and Endresen et al. [5]. The main feature of the present model is the timedependent variability of the cell volume $\mathfrak{v}_{i}$ since water molecules slowly diffuse across the cell membrane. Our model leads to an implicit dynamic link (the time derivative of the cell volume is a function of the transmembrane potential) 
between the cell volume, the transmembrane voltage potential, and the ionic concentrations. In the case of spherical cell, we explicit this link and perform numerical simulations.

Such a dynamic relationship between the cell volume and the transmembrane potential has not yet, to the best of our knowledge, been investigated. Three main results are presented in this paper: the new cell model (which takes the water diffusion into account), the explicit link between the cell volume and the transmembrane potential, and the explicit expression of the potential in terms of ionic concentrations.

For the sake of simplicity, we omit the contribution of active channels such as $\mathrm{Na}^{+} / \mathrm{K}^{+}$pumps and voltage-dependent ionic channels. These more accurate descriptions of the ionic fluxes can be added to our model with a very slight modification and without affecting the modelling principle. Our model can already provide some insight in specific mechanisms such as regulation of muscle fatigue. Osmotically induced cell swelling has been observed during muscle contraction [23, while muscle fatigue is associated with membrane depolarization [15, 2. Our model represents an integration of these results.

In section 2, we derive the ordinary differential equations that describe the transmembrane potential in terms of ionic concentrations and cell volume. Section 3 is devoted to the numerical simulations of the equations. In Section 4 , experiments that corroborate the numerical results are presented and we concluded by discussion.

\section{The transmembrane potential}

According to its phospholipid composition, the cell membrane is a resistive medium with a capacitance $C_{m}$ in F.m ${ }^{-2}$. We denote by $\mathfrak{S}_{\text {ions }}$ the group of ionic species. For our numerical simulations, $\mathfrak{S}_{\text {ions }}$ is $\left\{\mathrm{Na}^{+}, \mathrm{Cl}^{-}, \mathrm{K}^{+}, \mathrm{X}^{-}\right\}$, where $X^{-}$denotes the whole of the other ions that are considered non-permeant. This set is globally negatively charged on account of the excess $\mathrm{Na}^{+}$and $\mathrm{K}^{+}$inside the cell, and the mean charge valency of $X^{-}$equals -1.6 according to Fraser and Huang [7] and the references therein.

The membrane is sprinkled over with ionic channels, across which ionic exchanges occur. The valency of the ions $S$ is denoted by $z_{S}$, and the constant $P_{S}$ denotes the membrane permeability to the species $S$. In the following, $R$ and $F$ denote respectively the gas constant and the Faraday constant, and the temperature is denoted by $T$. Glossary of Table 1 summarizes the notations used in the paper.

\subsection{Ion flux through channels}

According to the literature [1, 3, 5, 13, the ionic fluxes depend on the transmembrane potential $V_{m}$. We use the convention $V_{m}=V_{i}-V_{o}$, where the indices $i$ and $o$ hold respectively for inner and outer part of the membrane. The ion flux equation previously described in 1943 by Goldman 9, and modified in 1949 by Hodgkin and Katz [11, is based on a Gas-Law model. It is precisely described in the book of Malmivuo and Plonsey 19, and in the article of Endresen et al. [5] (see also Armstrong [1]). According to these models, the current $I_{S}$ of the 
Table 1: Glossary

gas constant

Faraday constant

temperature

equal to $F /(2 R T)$

cell volume

membrane area

cell capacitance

transmembrane potential

membrane penetrability to water

equal to $R T \tau_{w}$

$\mathfrak{S}_{\text {ions }} \quad$ group of all the considered ionic species

$S \quad$ specific ionic species belonging to the group $\mathfrak{S}_{\text {ions }}$

$[S]_{e} \quad$ extracellular concentration of ions $S$

$[S]_{i} \quad$ cell concentration of ions $S$

$n_{S}^{i}$

$P_{S}$

amount of substance of ions $S$ in the cell

membrane permeability to ions $S$ valency of $S$

$z_{S}$

$I_{S}$

$g_{S}$

current due to the fluxes of ions $S$

conductance for ions $S$

$V_{S} \quad$ Nernst equilibrium potential of the ions $S$

$\Sigma_{e} \quad$ total sum of outer concentrations

$\Sigma_{i} \quad$ total sum of inner concentrations 
permeant ion $S$ equals:

$$
I_{S}=\frac{1}{2} z_{S} P_{S} F\left([S]_{i} e^{z_{S} c V_{m}}-[S]_{e} e^{-z_{S} c V_{m}}\right),
$$

where $c=F /(2 R T)$. The Nernst equilibrium potential $V_{S}$ of the species $S$ is the potential such that $I_{S}$ vanishes:

$$
V_{S}=\frac{1}{2 c z_{S}} \log \left(\frac{[S]_{e}}{[S]_{i}}\right)
$$

Let $g_{S}$ be the membrane conductance for the species $S$ defined by

$$
g_{S}=P_{S} z_{S}^{2} c F \sqrt{[S]_{e}[S]_{i}},
$$

we therefore infer

$$
I_{S}=g_{S} \frac{\sinh \left(z_{S} c\left(V_{m}-V_{S}\right)\right)}{z_{S} c} .
$$

When $z_{S} c\left(V_{m}-V_{S}\right)$ is small compared to 1 , equation (3) leads to the linear equation

$$
I_{S}=g_{S}\left(V_{m}-V_{S}\right)
$$

\subsection{Evolution of the ionic concentrations}

The global amount of ions driven by the total current of ions $S \in \mathfrak{S}_{\text {ions }}$ across the membrane $\Gamma$ equals

$$
\frac{\mathrm{d} n_{S}^{i}}{\mathrm{~d} t}=-\frac{\mathscr{A}(\Gamma)}{z_{S} F} I_{S},
$$

where $\mathscr{A}(\Gamma)$ denotes the membrane surface area.

\subsection{Osmolarity}

We denote by $\Sigma_{e}$ and $\Sigma_{i}$ the respective sums of the outer and inner concentrations

$$
\Sigma_{e}=\sum_{S \in \mathfrak{S}_{i o n s}}[S]_{e}, \quad \Sigma_{i}=\sum_{S \in \mathfrak{S}_{i o n s}} n_{S}^{i} / \mathfrak{v}_{i}
$$

Since the water is incompressible, we suppose that the change of the cell volume is described by the rate of flow of water across the membrane. Let $\tau_{w}$ be the water membrane penetrability ${ }^{1}$ in s.m ${ }^{-1}$ in S.I. units. We denote by $\Theta_{w}$ the following coefficient

$$
\Theta_{w}=R T \tau_{w} .
$$

According to the Landahl model [16] we infer

$$
\frac{\mathrm{d} \mathfrak{v}_{i}}{\mathrm{~d} t}=-\Theta_{w} \mathscr{A}(\Gamma)\left(\Sigma_{e}-\Sigma_{i}\right),
$$

\footnotetext{
${ }^{1}$ The parameter $\tau_{w}$ is a penetrability hence it is homogeneous to the inverse of a velocity. We refer to Landalh [16] for more precision.
} 
Recently, Hernández and Cristina [10, or Mathai et al. 20] replaced $\Theta_{w}$ by the product of the membrane permeability $P_{w}$ by the partial molar volume of water $\mathscr{V}_{w}$. Therefore the coefficients $\tau_{w}$ and $P_{w}$ are linked by the following equality:

$$
\tau_{w}=\frac{P_{w} \mathscr{V}_{w}}{R T} .
$$

Remark 2.1. In many models [1, 5, 7, 19], the plasma membrane cannot support a pressure difference. Therefore osmotic equilibrium is required at any time

$$
\Sigma_{e}=\Sigma_{i}, \quad \text { which leads to } \mathfrak{v}_{i}=\frac{1}{\Sigma_{e}} \sum_{S \in \mathfrak{S}_{\text {ions }}} n_{i}^{S} .
$$

This equality enforces the volume to change instantaneously when the osmotic equilibrium is modified. However, the cell volume is adjusted by the diffusion of water molecules across the membrane. This phenomenon takes time and is described by the model of Landahl [16], which is based on Fick's law type model to describe the diffusion of water molecules through the membrane.

Remark 2.2. Note that Mathai et al. [20] write the volume change [6] in a different way. Let $R_{\text {vol }}$ be the ratio of the volume $\mathfrak{v}_{i}$ at the time $t$ divided by the initial volume $\mathfrak{v}_{i}^{0}$, and denote by $\Sigma_{i}^{0}$ the sum of the initial inner concentrations. Mathai et al. suppose that cell membrane is perfectly non permeant. Since no exchange occurs inner and outer amounts of substance of the ionic species are constant. Therefore

$$
\Sigma_{i}=\Pi_{i}^{0} / R_{v o l},
$$

and we infer the Mathai et al. equation:

$$
\frac{\mathrm{d} R_{v o l}}{\mathrm{~d} t}=-\Theta_{w} \frac{\mathscr{A}(\Gamma)}{\mathfrak{v}_{i}^{0}}\left(\Sigma_{e}-\frac{\Pi_{i}^{0}}{R_{v o l}}\right) .
$$

However, we emphasize that we cannot consider the inner amounts of substance constant since the ionic fluxes drive ions across the membrane. Therefore equation (6) must be used. Observe that equation (6) leads to (7) by considering that no ionic flux across the membrane occurs.

\subsection{Equations for the transmembrane potential}

Our model links the transmembrane potential, the cell volume, and the ionic concentrations inside and outside the cell. The unknown quantities are the transmembrane potential $V_{m}$, the cell volume $\mathfrak{v}_{i}$, and the amount in the cell of substances $n_{S}^{i}$ of the ions $S \in \mathfrak{S}_{\text {ions }}$. We obtain a system of ordinary differential equations. This system is very similar to the models of Endresen et al. [5] and Armstrong [1. The main difference consists in the fact that the cell volume evolution is governed by an ordinary differential equation while Endresen et al. and Armstrong et al. required osmotic equilibrium at any time. Using the 
Kirchoff law and the above equalities we infer

$$
\begin{aligned}
& C_{m} \frac{\mathrm{d} V_{m}}{\mathrm{~d} t}+\sum_{S \in \mathfrak{S}_{i o n s}} I_{S}=0, \\
& I_{S}=g_{S} \frac{\sinh \left(z_{S} c\left(V_{m}-V_{S}\right)\right)}{z_{S} c}, \quad \text { for } S \in \mathfrak{S}_{i o n s}, \\
& \frac{\mathrm{d} n_{S}^{i}}{\mathrm{~d} t}=-\frac{\mathscr{A}(\Gamma)}{z_{S} F} I_{S}, \\
& \frac{\mathrm{d} \mathfrak{v}_{i}}{\mathrm{~d} t}=-\Theta_{w} \mathscr{A}(\Gamma)\left(\Sigma_{e}-\Sigma_{i}\right),
\end{aligned}
$$

where

$$
\left\{\begin{array}{l}
{[S]_{i}=n_{S}^{i} / \mathfrak{v}_{i}, \quad V_{S}=\frac{1}{2 z_{S} c} \log \left([S]_{e} /[S]_{i}\right), \quad g_{S}=z_{S}^{2} P_{S} c F \sqrt{[S]_{e} n_{S}^{i} / \mathfrak{v}_{i}}} \\
\Sigma_{e}=\sum_{S \in \mathfrak{S}_{\text {ions }}}[S]_{e}, \quad \Sigma_{i}=\sum_{S \in \mathfrak{S}_{i o n s}}[S]_{i}
\end{array}\right.
$$

For the linearized model we replace $8 \mathrm{~b}$ by

$$
I_{S}=g_{S}\left(V_{m}-V_{S}\right) \text {. }
$$

The novelty of the model consists in equation (8d), which describes the evolution of the cell volume. To solve problem (8), initial conditions are chosen for $V_{m}$, $\mathfrak{v}_{i}$, and concentrations $n_{S}^{i}$.

\subsection{Explicit link between the cell radius and the potential}

Problem (8) provides an implicit link between the cell volume and the transmembrane potential through the ionic concentrations and the ionic fluxes. The aim of the paragraph is to write explicitly this link. Equation 8d can be rewritten as follows

$$
\frac{1}{\Theta_{w}} \frac{\mathfrak{v}_{i}}{\mathscr{A}(\Gamma)} \frac{\mathrm{d} \mathfrak{v}_{i}}{\mathrm{~d} t}=-\Sigma_{e} \mathfrak{v}_{i}+\sum_{S \in \mathfrak{S}} n_{S}^{i}
$$

Differenciating the above equality and using equation $8 \mathrm{c}$ leads to

$$
\frac{1}{\Theta_{w}} \frac{\mathrm{d}}{\mathrm{d} t}\left(\frac{\mathfrak{v}_{i}}{\mathscr{A}(\Gamma)} \frac{\mathrm{d} \mathfrak{v}_{i}}{\mathrm{~d} t}\right)=-\Sigma_{e} \frac{\mathrm{d} \mathfrak{v}_{i}}{\mathrm{~d} t}-\frac{\mathscr{A}(\Gamma)}{F} \sum_{S \in \mathfrak{S}} \frac{1}{z_{S}} I_{S}
$$

Considering the linearized model for the ionic fluxes we therefore infer the explicit link between the shape of the cell and the transmembrane potential:

$$
\frac{1}{\Theta_{w}} \frac{\mathrm{d}}{\mathrm{d} t}\left(\frac{\mathfrak{v}_{i}}{\mathscr{A}(\Gamma)} \frac{\mathrm{d} \mathfrak{v}_{i}}{\mathrm{~d} t}\right)=-\Sigma_{e} \frac{\mathrm{d} \mathfrak{v}_{i}}{\mathrm{~d} t}-\frac{\mathscr{A}(\Gamma)}{F} \sum_{S \in \mathfrak{S}} \frac{g_{S}}{z_{S}}\left(V_{m}-V_{S}\right)
$$

Observe that the above equation involves both cell volume and membrane area. To uniquely determine the shape of the cell it is necessary to add a law on the membrane surface. However, in the experiments, the cells in suspension are almost spherical and therefore the geometry is entirely determined by the cell radius. The next paragraph is devoted to give the explicit link between the transmembrane potential and the cell radius. 


\section{5.a The case of spherical cells}

Let us now consider spherical cells. Equation (8d) leads to the following equation on the cell radius $r$ :

$$
\frac{1}{\Theta_{w}} \frac{\mathrm{d} r}{\mathrm{~d} t}=-\left(\Sigma_{e}-\sum_{S \in \mathfrak{S}} \frac{n_{S}^{i}}{4 \pi r^{3} / 3}\right)
$$

Using equation (8c) with the linearized model of the ionic fluxes, and by differenciation we obtain a non-linear differential equation on $r$ :

$$
\frac{1}{\Theta_{w}} \frac{1}{3 r^{2}} \frac{\mathrm{d}}{\mathrm{d} t}\left(r^{3} \frac{\mathrm{d} r}{\mathrm{~d} t}\right)=-\Sigma_{e} \frac{\mathrm{d} r}{\mathrm{~d} t}-\sum_{S \in \mathfrak{S}_{i o n s}} \frac{g_{S}}{z_{S} F}\left(V_{m}-V_{S}\right) .
$$

The initial conditions are then :

$$
\left.r\right|_{t=0}=r_{0},\left.\quad \frac{\mathrm{d} r}{\mathrm{~d} t}\right|_{t=0}=-\Theta_{w}\left(\Sigma_{e}-\Sigma_{i}^{0}\right) .
$$

Denote by $V_{m}^{\text {rest }}$ the resting transmembrane potential defined such that:

$$
\sum_{S \in \mathfrak{S}_{i o n s}} \frac{g_{S}}{z_{S}}\left(V_{m}^{r e s t}-V_{S}\right)=0
$$

If the cell is initially in osmotic equilibrium and if $V_{m}$ equals $V_{m}^{r e s t}$ then equation (11) shows that $r$ remains at its initial value. However, a slight change in the value of $V_{m}$ leads to an increase (or a decrease) of the cell radius.

\subsection{Explicit formula for the transmembrane potential.}

It is interesting to obtain an explicit expression of the transmembrane potential in terms of the ionic concentrations. This can be achieved by assuming that the cell shape does not change. Actually if the cell volume $\mathfrak{v}_{i}$ and the membrane area $\mathscr{A}(\Gamma)$ are constant $-i . e$. the osmotic equilibrium is reached-then using equality $8 \mathrm{c}$, the equation $8 \mathrm{a}$ writes

$$
C_{m} \frac{\mathrm{d} V_{m}}{\mathrm{~d} t}=\frac{\mathfrak{v}_{i}}{\mathscr{A}(\Gamma)} F \sum_{S \in \mathfrak{S}} z_{S} \frac{\mathrm{d}[S]_{i}}{\mathrm{~d} t} .
$$

Denoting by $V_{m}^{0}$ and $[S]_{i}^{0}$, the initial potential and inner concentrations respectively, we infer the explicit formula for the potential in terms of the inner concentrations at each time $t$ :

$$
\left(V_{m}-V_{m}^{0}\right)=\frac{\mathfrak{v}_{i}}{\mathscr{A}(\Gamma)} \frac{F}{C_{m}} \sum_{S \in \mathfrak{S}} z_{S}\left([S]_{i}-[S]_{i}^{0}\right) .
$$

Define the initial potential by

$$
V_{m}^{0}=\frac{\mathfrak{v}_{i}}{\mathscr{A}(\Gamma)} \frac{F}{C_{m}} \sum_{S \in \mathfrak{S}} z_{S}[S]_{i}^{0}
$$


Then, the following formula, that is similar to the "Charge Difference" equation of Fraser and Huang holds

$$
V_{m}=\frac{\mathfrak{v}_{i}}{\mathscr{A}(\Gamma)} \frac{F}{C_{m}} \sum_{S \in \mathfrak{S}} z_{S}[S]_{i} .
$$

Observe that the factor $\mathfrak{v}_{i} / \mathscr{A}(\Gamma)$ ensures the dimensional consistency of the previous equality. It is striking that equation 12 involves only the inner concentrations, while the concentrations of the exterior solution is expected to have an influence on the potential. This is hidden behind the hypothesis that the outer concentrations are constant, and by supposing that this equality (13) holds at the initial time. Actually, if the outer concentrations change, using the mass conservation law, we would have:

$$
\frac{\mathrm{d} n_{S}^{e}}{\mathrm{~d} t}=-\frac{\mathrm{d} n_{S}^{i}}{\mathrm{~d} t} .
$$

Therefore the expression 12 of the potential would be:

$$
\left(V_{m}-V_{m}^{0}\right)=\frac{F}{2 C_{m}} \frac{\mathfrak{v}_{i}}{\mathscr{A}(\Gamma)} \sum_{S \in \mathfrak{S}} z_{S}\left(\left([S]_{i}-[S]_{e}\right)-\left([S]_{i}^{0}-[S]_{e}^{0}\right)\right),
$$

and then supposing that

$$
V_{m}^{0}=\frac{F}{2 C_{m}} \frac{\mathfrak{v}_{i}}{\mathscr{A}(\Gamma)} \sum_{S \in \mathfrak{S}} z_{S}\left([S]_{i}^{0}-[S]_{e}^{0}\right)
$$

we would have

$$
V_{m}=\frac{F}{2 C_{m}} \frac{\mathfrak{v}_{i}}{\mathscr{A}(\Gamma)} \sum_{S \in \mathfrak{S}} z_{S}\left([S]_{i}-[S]_{e}\right) .
$$

This formula is exactly the Endresen et al. formula (63) of [5]: just replace the factor $C_{m} \mathscr{A}(\Gamma)$ by the factor $C$ of 5 . Observe that Endresen et al. consider $C$

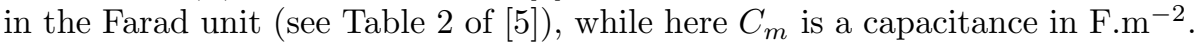
Therefore $C$ and $C_{m} \mathscr{A}(\Gamma)$ are homogeneous. Equation (14) is a generalization of the result of Endresen et al.

\section{Numerical simulations}

We perform numerical simulations of the linearized problem for the case of spherical cells. For all simulations, the initial radius equals $10 \mu \mathrm{m}$. The numerical parameters are given in Table 2

Observe that the constant $1 / C_{m}$ is quite large since $C_{m} \sim 5.10^{-2}$ S.I. Therefore a steady-state is reached faster by the transmembrane potential than by the cell radius and the ionic concentrations. To solve the ordinary differential equations of the model we use the $4^{\text {th }}$ order Runge-Kutta scheme. This scheme has the advantage of being explicit (thus simple to implement) and at the same time quite stable compared with the usual Euler explicit scheme. 
Table 2: Numerical parameters

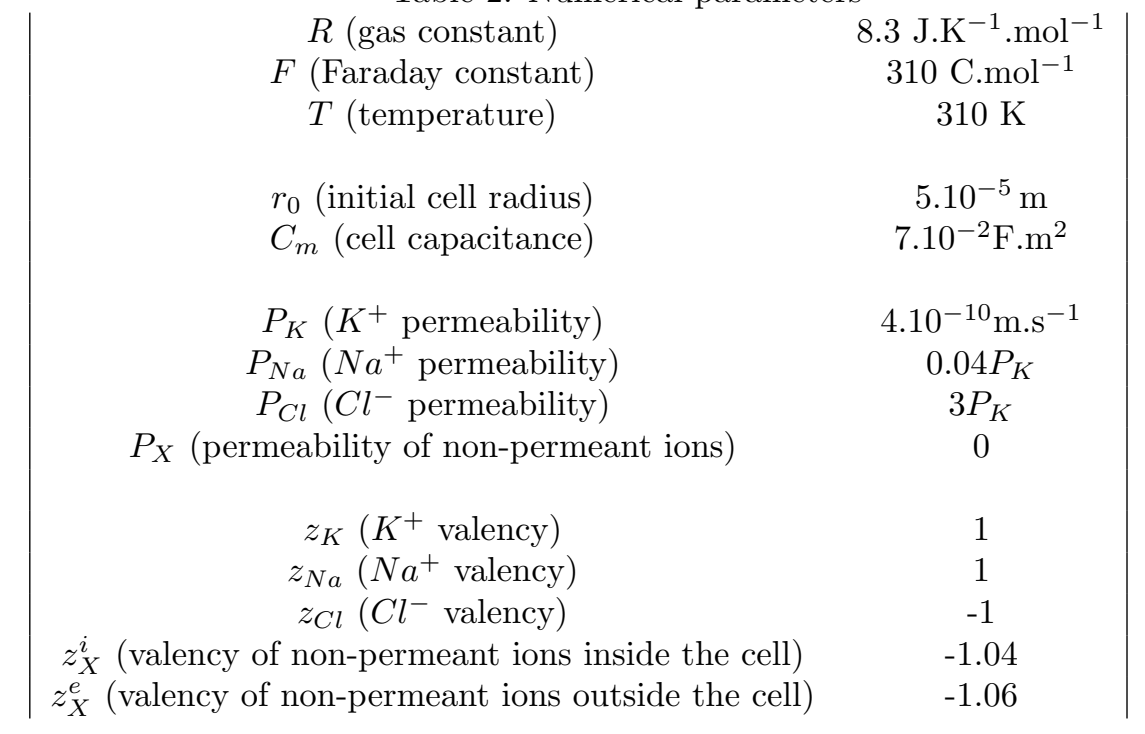

\subsection{Effect of an osmotic stress on the transmembrane po- tential and the cell volume.}

The value of $\tau_{w}$ is fixed at $10^{-12} \mathrm{~s}^{-1}$ as given by Landahl [16, which corresponds to a water permeability $P_{w}$ of $10^{-5} \mathrm{~m} \cdot \mathrm{s}^{-1}$ (see Mathai et al. [20]). According to Lodish et al. [18, the inner and outer ionic concentrations for the mammalian cell in osmotic equilibrium are

$$
\begin{aligned}
& {[N a]_{i}=12 \mathrm{mM}, \quad[K]_{i}=139 \mathrm{mM}, \quad[C l]_{i}=4 \mathrm{mM}, \quad[X]_{i}=141 \mathrm{mM},} \\
& {[N a]_{e}=145 \mathrm{mM}, \quad[K]_{e}=4 \mathrm{mM}, \quad[C l]_{e}=116 \mathrm{mM}, \quad[X]_{e}=31 \mathrm{mM},}
\end{aligned}
$$

and we imposed the values of the ions permeabilities and valencies given by Fraser and Huang [7] as ${ }^{2}$

$$
\begin{aligned}
& P_{K}=4.10^{-10} \mathrm{~m} . \mathrm{s}^{-1}, \quad P_{N a}=0.04 P_{K}, \quad P_{C l}=3 P_{K}, \quad P_{X}=0 \\
& z_{N a}=z_{K}=1, \quad z_{C l}=-1, \quad z_{X}^{i}=-1.04, \quad z_{X}^{e}=-1.06
\end{aligned}
$$

For such concentrations, the resting transmembrane potential for a cell of radius $10 \mu \mathrm{m}$ equals $-88 \mathrm{mV}$. We imposed this value as the initial condition for the transmembrane potential.

The simulations deal with the effect of an osmotic stress both on transmembrane potential and cell volume. The osmotic stress is applied at $t=0$, and we let the system evolve. For the hypertonic trial, the outer osmotic pressure of the solution is set at $456 \mathrm{mM}$, while for the hypotonic trial, we increase the inner osmotic pressure to $456 \mathrm{mM}$. For both cases we investigate three types of

\footnotetext{
${ }^{2}$ The valencies $z_{X}^{i}$ and $z_{X}^{e}$ are the respective mean valencies of the non-permeant ions inside and outside the cell in order to ensure the electroneutrality of both outer and inner media. Since the non-permenant ions inside and outside the cell are different, then their respective mean valencies differ also.

${ }^{3}$ Observe that $-88 \mathrm{mV}$ is the resting membrane potential obtained by Fraser and Huang (Table 2 page 466 in [7]) for similar concentrations.
} 
osmotic stresses: first we add non-permeant ions $X^{-}$, then study the effect of an increase in $\mathrm{NaCl}$ concentrations and then do the same for $\mathrm{KCl}$.

\section{1.a Hyperosmotic stress}

In order to study an hypertonic stress, we increase the outer osmolarity by 150 $\mathrm{mM}$ (from $296 \mathrm{mM}$ to $446 \mathrm{mM}$ ). This stress is simulated in three ways: addition of non-permeant ions $X\left([X]_{e}=181 \mathrm{mM}\right)$, addition of $\mathrm{NaCl}\left([\mathrm{Na}]_{e}=220 \mathrm{mM}\right.$, $\left.[C l]_{e}=191 \mathrm{mM}\right)$, and addition of $\mathrm{KCl}\left([\mathrm{K}]_{e}=79 \mathrm{mM},[C l]_{e}=191 \mathrm{mM}\right)$, while the other concentrations remain unchanged. Fig. 1(b) gives the evolution of the cell volume for the three stresses. We observe the coincidence of the three curves, meaning that cell volume regulation is independent of the osmotic stress type.

The stabilization delay and change in volume obtained with the model correspond to experimental results presented by Ferencziet al. (Fig 4.B, pp 429 6]). On Fig. 1(a), we observe that the value of the transmembrane potential is highly dependent on the stress type. The value of the steady potential is around -90 $\mathrm{mV}$ when the stress is achieved by adding $\mathrm{X}$ ions or $\mathrm{NaCl}$, whereas the potential changes dramatically and raises up to $-50 \mathrm{mV}$ when $\mathrm{KCl}$ is added. Stabilization of the transmembrane potential occurs within few seconds for the three stresses, while cell volume stabilization is achieved much later, as expected.

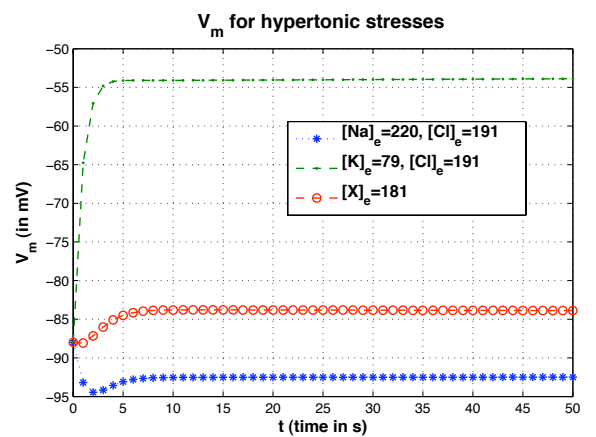

(a) Potential regulation

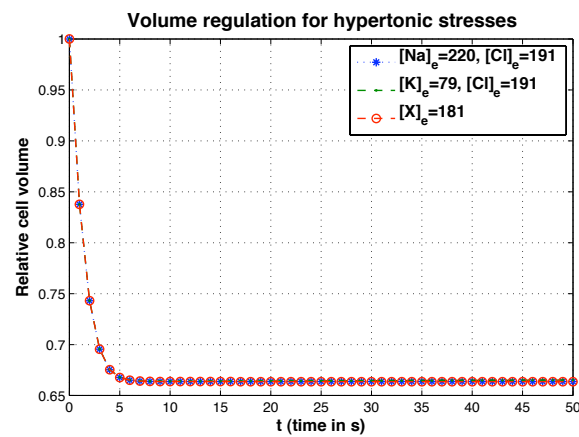

(b) Volume regulation

Figure 1: Transmembrane potential (Fig. 1(a) and cell volume (Fig. 1(b)) responses to a hypertonic stress: $P_{e}=446 \mathrm{mM}, P_{i}=296 \mathrm{mM}$. The water penetrability equals $\tau_{w}=10^{-12}{\mathrm{~s} . \mathrm{m}^{-1}}$.

\section{1.b Hypo-osmotic stress}

We then study hypotonic stress, increasing the inner osmolarity by $150 \mathrm{mM}$ (from $296 \mathrm{mM}$ to $446 \mathrm{mM}$ ). Here again, the stress is simulated in three ways: addition of non-permeant ions $X\left([X]_{i}=291 \mathrm{mM}\right)$, addition of $\mathrm{NaCl}\left([\mathrm{Na}]_{i}=\right.$ $\left.87 \mathrm{mM},[C l]_{i}=79 \mathrm{mM}\right)$, and addition of $\mathrm{KCl}\left([\mathrm{K}]_{i}=219 \mathrm{mM},[C l]_{i}=74\right.$ $\mathrm{mM}$ ), other concentrations being unchanged. The evolution of the cell volume for the three stresses is displayed in Fig. 2(b). We observe that the three curves coincide, which means that the cell volume regulation is independent of the three types of osmotic stress, similarly to the hypertonic stresses. However, 
the 3 minutes delay required for the cell volume stabilization is much larger than for the hypertonic trial. On Fig. 2(a) we observe that the value of the transmembrane potential is highly dependent on the stress type: it stabilizes around $-30 \mathrm{mV}$ when the stress is produced by addition of $\mathrm{X}$ ions or $\mathrm{NaCl}$, whereas it dramatically lowers down to $-90 \mathrm{mV}$ with the addition of $\mathrm{KCl}$. The transmembrane potential stabilizes within few seconds for the three stresses as for the hyperosmotic stresses.

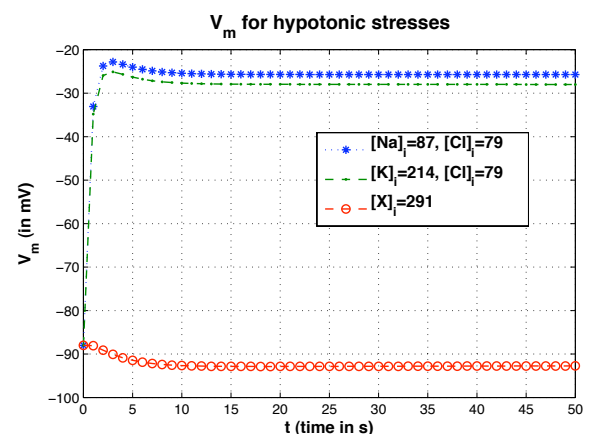

(a) Potential regulation

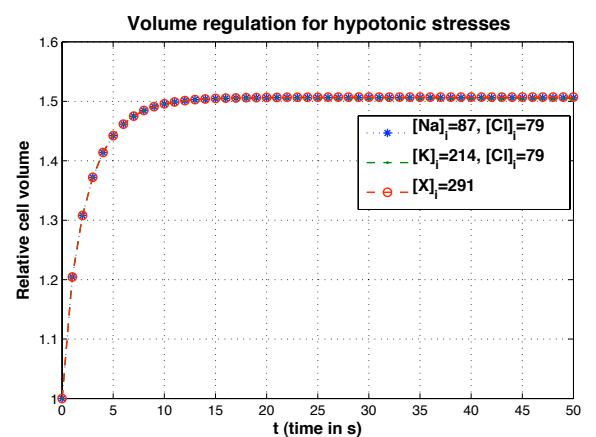

(b) Volume regulation

Figure 2: Transmembrane potential (Fig. 2(a) and cell volume (Fig. 2(b) behaviours for hypertonic solution: $P_{e}=296 \mathrm{mM}, P_{i}=446 \mathrm{mM}$. The water penetrability equals $\tau_{w}=10^{-12} \mathrm{~s} . \mathrm{m}^{-1}$.

\section{1.c Influence of the coefficient $\tau_{w}$ on the dynamics}

In this paragraph we study the effect of a reduction in water penetrability $\tau_{w}$ on the dynamics of the phenomena. Note the difference between the coefficient $\tau_{w}$ and the coefficient of permeability, since S.I. units for penetrability are s.m ${ }^{-1}$ and $\mathrm{m} \cdot \mathrm{s}^{-1}$ for permeability. The coefficient $\tau_{w}$ describes the time taken by a water molecule to cross the membrane: the higher $\tau_{w}$, the more permeant the membrane is. In accordance with Landahl [16], $\tau_{w}$ usually equals $10^{-12} \mathrm{~s} . \mathrm{m}^{-1}$. We numerically investigate the effect of a reduction of this value. According to Fig. 3 when the membrane penetrability to water $\tau_{w}$ decreases from $10^{-12}$ $\mathrm{s} . \mathrm{m}^{-1}$ to $10^{-14} \mathrm{~s} . \mathrm{m}^{-1}$, a delay in the stabilization of the cell volume can be observed. Actually, while the stabilization occurs in a few seconds for $\tau_{w}=$ $10^{-12} \mathrm{~s} . \mathrm{m}^{-1}$, the cell volume reaches its steady state within a minute when

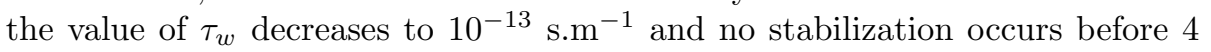
$\min$ for $\tau_{w}=10^{-14}$. Compared with the experiments of Rouzaire-Dubois et al. [21, 4, 22], the value of the stabilized cell volume is plausible. A decrease of $\tau_{w}$, caused by alterations of the membrane phospholipids for instance, could explain the differences in the delays experimentally observed by Rouzaire-Dubois et al. [4, 21] in the cell osmotic stabilization. Moreover let us note that the values of the steady potential given by Fig. 4 are similar (around $-90 \mathrm{mV}$ ) for $\tau_{w}$ equals $10^{-12}, 10^{-13}$ or $10^{-14}$, meaning that a decrease of the penetrability of water does not affect the value of the transmembrane potential but dynamics of the volume regulation. 


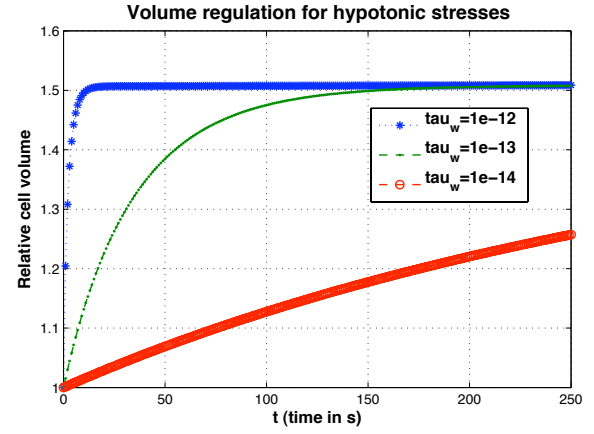

(a) Hypotonic stress

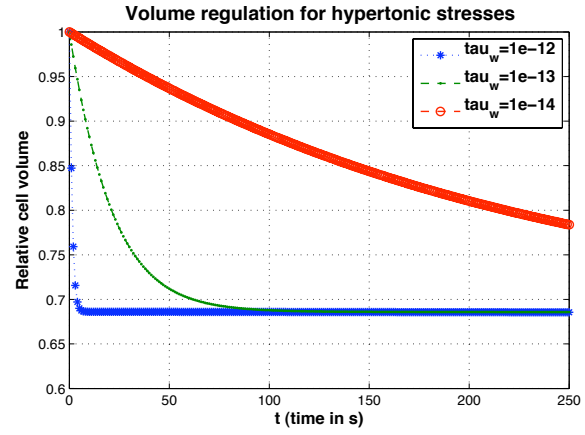

(b) Hypoternic stress

Figure 3: Cell volume behaviour for hypotonic (Fig. 3(a) and hypertonic (Fig. 3(b) stresses of amplitude $150 \mathrm{mM}$ by changing the concentration of non-permeant ions $X$.

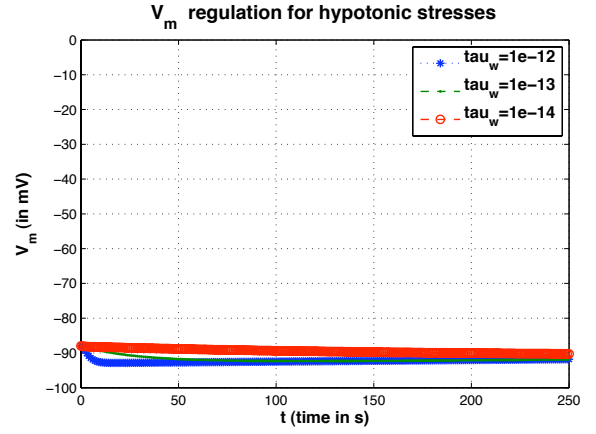

(a) Hypotonic stress

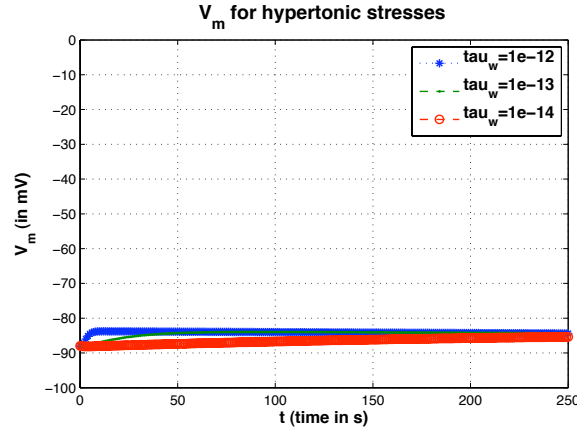

(b) Hypertonic stress

Figure 4: Transmembrane potential behaviours for hypotonic (Fig. 4(a)) and hypertonic (Fig. 4(b) stresses of amplitude $150 \mathrm{mM}$ by changing the concentration of non-permeant ions $X$.

\section{Preliminary experiments}

In order to validate the theoretical results we performed preliminary experiments. More precisely in this section, we aim at showing that the dynamic of the experimental cell volume stabilization occurs within the time predicted by the model after osmotic stresses.

\subsection{Materials and Methods}

\section{1.a Cell culture}

DC-3F cells (Chinese hamster fibroblast lung cells) were grown in Minimum Essential Medium (Invitrogen, Cergy-Pontoise, France) supplemented with 10\% fetal bovine serum (Invitrogen), $500 \mathrm{U} / \mathrm{ml}$ penicillin, $500 \mu \mathrm{g} / \mathrm{ml}$ streptomycin (Invitrogen) defined as complete medium. Cultures were maintained in a hu- 
midified atmosphere with $5 \% \mathrm{CO}_{2}$ at $37^{\circ} \mathrm{C}$. Cells were routinely passed every two days.

\section{1.b Solutions used}

The solutions used were all PBS based (D-PBS 10x, Invitrogen, diluted with milli-Q water, final molarities : $\mathrm{Na}^{+}-154 \mathrm{mM} ; \mathrm{K}^{+}-4 \mathrm{mM} ; \mathrm{Cl}^{-}-140 \mathrm{mM}$ ; $\mathrm{H}_{2} \mathrm{PO}_{4}^{-}-1,5 \mathrm{mM} ; \mathrm{HPO}_{4}^{2-}-8 \mathrm{mM}$ ). Hypertonic solutions were obtained by adding either $\mathrm{NaCl}$ or $\mathrm{KCl}$ in order to increase their concentration by a minimum of $25 \mathrm{mM}$ and a maximum of $300 \mathrm{mM}$.

\section{1.c Fluorescence Activated Cell Sorter (FACS) experiments}

Cells were harvested by trypsin and placed in a PBS solution for 15 minutes before any osmotic stress. The hypertonic stresses were obtained by adding the suitable volume of PBS supplemented with $300 \mathrm{mM} \mathrm{NaCl}$ or $\mathrm{KCl}$ to $500 \mu \mathrm{L}$ of the PBS containing the cells in suspension. In FACS analysis, forward scattering (FSC) was used to estimate the final volume ratios. Approximately 50.000 cells were run through the FACS three minutes after the hypertonic stresses. The measure lasted $40 \mathrm{~s}$ during which the FSC was stable. The dynamic evolution of the FSC was obtained starting with $800 \mu \mathrm{L}$ of cells in suspension. $300 \mu \mathrm{L}$ of those were used to obtain the initial FSC value. The tube was then removed from the FACS and replaced with another one containing the remaining $500 \mu \mathrm{L}$ mixed with the right volume of PBS supplemented with $\mathrm{NaCl}$ solution at 300 $\mathrm{mM}$ just before starting the stressed cells FACS acquisition. Each reported value is the average of 100 to 500 cells FSC acquisition.

\subsection{Statistical analysis of FSC measurements}

In static experiments, since the cells populations did not follow a Gaussian distribution, the mean FSC for each individual experiment is the average value of all the events considered as cells. Final results are the average of three individual experiments \pm standard deviation (SD).

\subsection{Results}

For different hypertonic solutions, cells were analyzed using a FACS three minutes after the hypertonic stress. The mean FSC, which can be considered as a good representation of cells size, was measured and experimental volume ratios were thus calculated. Simultaneously numerical simulations of the model are performed with the experimental values of the initial concentrations. Experimental and numerical ratios are plotted on Fig. 5. It appears that theoretical values are very close to experimental data even though they are slightly under estimated for the hypertonic stress obtained by addition of $\mathrm{KCl}$. Noticeably, for the same increase in molarity, the model predicts a lower amplitude of the volume change after $\mathrm{KCl}$ than $\mathrm{NaCl}$ stress. Experimental data confirm this tendency but show a difference more important than the one predicted.

The dynamic evolution of the FSC (as explained in Materials and Methods) was recorded for the three different $\mathrm{NaCl}$ stresses indicated on Fig. 5 . The experimental averaged data are plotted in thin line, the beginning of the stresses 


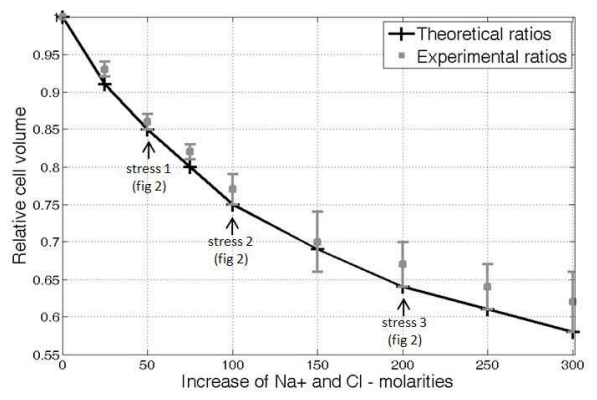

(a) Addition of $\mathrm{NaCl}$

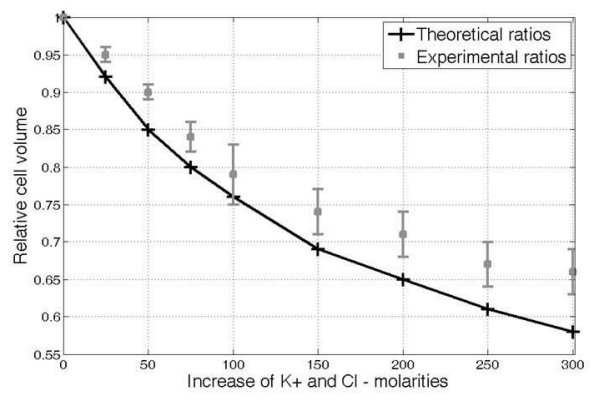

(b) Addition of $\mathrm{KCl}$

Figure 5: Relative cell volume after stabilization for an osmotic stress of 150 $\mathrm{mM}$ by adding respectively $\mathrm{NaCl}$ (Fig $5(\mathrm{a})$ ) and $K C l$ (Fig. $5(\mathrm{~b})$ ) (Means $\pm \mathrm{SD}$ of three experiments).

being voluntarily shifted of ten seconds each. The part of the curves at no change in cell volume (initial FSC) were acquired from a $300 \mu \mathrm{L}$ cell suspension. Then, the missing part of the curves corresponds to the time required for removing the tube from the FACS and replacing it with the cells just osmotically stressed. On Fig. 6w we have superimposed theoretical volume evolution for three different values of membrane penetrability to water denoted by $\tau_{w}$. The experimental data provided by FSC cannot be precisely fitted since Fig. 5 shows that the final volume ratio values differed from the prediction. Still, we can give a good framing of $\tau_{w}$ as all three stresses have their best fit for $\tau_{w}=7.10^{-13}{\mathrm{~s} . \mathrm{m}^{-1}}^{-}$ Stress 2 in particular validates the dynamic part of the model.

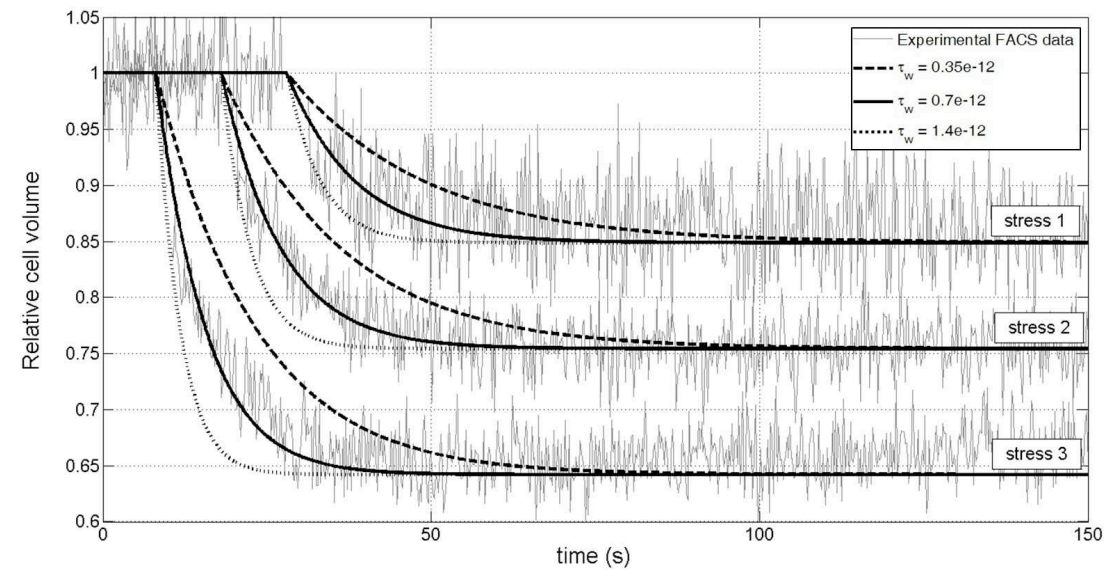

Figure 6: Dynamic evolution of the relative cell volume for the 3 stresses defined in the text. 


\section{Discussion}

We have proposed a complete and generic model for the transmembrane potential and cell volume regulation. This model, based on ordinary differential equations, links dynamically the membrane potential, the cell volume, and the internal and external ionic concentrations. The main insight consists in taking into account the water diffusion across the membrane due to the osmotic pressure. This leads to an ordinary differential equation of the cell volume with respect to ionic concentrations. In the case of spherical cells, we explicitly link the behaviour of the cell volume to the transmembrane potential.

Preliminary experiments validate the model proposed in this manuscript. The numerical simulations fit very well with the experimental data after $\mathrm{NaCl}$ addition, while a larger difference appears after $K C l$ addition. It can be noticed that the final values obtained during dynamic experiments are coherent with the ones obtained by static analysis of a large number of cells. Moreover, the time resolution of the FACS seems well adapted to this kind of dynamics. Indeed, the FACS is fast enough to describe quite precisely the volume evolution, the time needed to actually mix solutions in order to make the stress being the limiting factor. FACS thus appears as an efficient tool to evaluate the volume evolution dynamically. In a next step it will be interesting to investigate if the dynamics are different depending on the cell types. Indeed the differences between $\mathrm{KCl}$ and $\mathrm{NaCl}$ stresses might be relevant to metabolic issues. Using different cells types or acting on metabolism with appropriate drugs are avenues that appear worth exploring.

Our model can be derived to obtain the models presented previously by Jakobsson [14, Armstrong [1, Endresen et al. [5], Fraser and Huang [7, and Hernández [10]. More precisely, if the cell volume is constant, we recover the model of Endresen et al. [5], and if the osmotic equilibrium is instantly reached, our model is then similar to the model of Arsmtrong [1] and leads to the "Charge Difference" equation of Fraser and Huang [7. However, these two hypotheses cannot explain the delays in cell volume stabilization experimentally observed by Rouzaire-Dubois et al. 4, 22, for the fifth generation of cells in culture submitted to a permanent osmotic stress. Comparatively, the first generation stabilizes very quickly [4. Our model provides a possible explanation for such delays. Alterations of the membrane lipids, increasing with each new generation of cell culture, could decrease the value of $\tau_{w}$, thus leading to such results.

\section{Acknowledgements}

The authors thank very warmly Professor O. Lafitte from the Department of Mathematics of Université Paris 13 for his well-considered advices and suggestions.

This research was partially supported by the INRIA collaborative research initiative ARC C3MB. A. Silve is supported by a DGA grant.

\section{References}

[1] C. M. Armstrong. The $\mathrm{Na} / \mathrm{K}$ pump, $\mathrm{Cl}$ ion, and osmotic stabilization of cells. P.N.A.S, 100:6257-6262, 2003. 
[2] T. Clausen and O.B. Nielsen. Potassium, na,$+ \mathrm{k}+-$ pumps and fatigue in rat muscle. J Physiol., 584:295-304, 2007.

[3] K. DeBruin and W. Krassowska. Modelling electroporation in a single cell. I. Effects of field strength and rest potential. Biophysical Journal, 77:12131224, Sept 1999.

[4] J-M. Dubois and B. Rouzaire-Dubois. The influence of cell volume changes on tumour cell proliferation. Eur. J. Physiol., 33:227-232, 2004.

[5] L.P. Endresen, K. Hall, Høye J.S., and J. Myrheim. A theory of the membrane potential of living cells. Eur. Biophys. J., 29:90:103, 2000.

[6] E. A. Ferenczi, J. A. Fraser, S. Chawla, J.N. Skepper, and C. Schwiening, C.J. Huang. Membrane potential stabilization in amphibian skeletal muscle fibres in hypertonic solutions. J. Physiol., 555.2:423-438, 2003.

[7] J. A. Fraser and C. Huang. A quantitative analysis of cell volume and resting potential determination and regulation in excitable cells. J. Physiol., 559.2:459-478, 2004.

[8] J. A. Fraser and C. Huang. Quantitative techniques for steady-state calculation and dynamic integrated modelling of membrane potential and intracellular ion concentrations. Prog Biophys Mol Biol., 94:336-372, 2007.

[9] D.E. Goldman. Potential, impedance and rectification in membranes. J. General Physiology, 27:37-60, 1943.

[10] J.A. Hernández and E. Cristina. Modeling cell volume regulation in nonexcitable cells: the roles of the nat pump and of cotransport systems. Am. J. Physiol. Cell Physiol., 275:1067-1080, 1998.

[11] A.L Hodgkin and B. Katz. The effect of sodium ions on the electrical activity of the giant squid axon. J.Physiology, 108:37-77, 1949

[12] L. Hodgkin and P. Horowicz. The influence of potassium and chloride ions on the membrane potential of single muscle fibres. J. Physiol., 148:127-160, 1959 .

[13] L. Hodgkin and A. Huxley. A quantitative description of membrane current and its application to conduction and excitation in nerve. J. Physiol., 117:500-544, 1952.

[14] E. Jakobsson. Interactions of cell volume, membrane potential and membrane transport parameters. American Journal of Physiology, 238:C196206, 1980.

[15] C. Juel. Potassium and sodium shifts during in vitro isometric muscle contraction, and the time course of the ion-gradient recovery. PflugersArch., 406:458-463, 1986.

[16] H.D. Landahl. A note on the units of membrane permeability to water. Bulletin of Mathematical Biophysics, 10, 1948. 
[17] F. Lang, G.L. Busch, M. Ritter, H. Völkl, S. Waldegger, E. Gulbins, and D. Häussinger. Functional significance of cell volume regulatory mechanisms. Physiol Rev., 78:247-306, 1998.

[18] H. Lodish, A. Berk, L.S. Zipursky, P. Matsudaira, D. Baltimore, and Darnell J. Molecular Cell Biology. Fourth Edition. W.H. Freeman and company, New York, 1986.

[19] J. Malmivuo and R. Plonsey. Bioelectromagnetism-Principles and Applications of Bioelectric and Biomagnetic Fields. Oxford University Press, New York, 1995.

[20] J.C. Mathai, S. Tristram-Nagle, J.F. Nagle, and M.L. Zeidel. Structural determinants of water permeability through the lipid membrane. The Journal of General Physiology, 131(1):69-76, 2007.

[21] B. Rouzaire-Dubois and J-M. Dubois. $\mathrm{K}^{+}$channel block-induced mammalianthe influence of cell volume changes on tumour cell proliferation. Eur. J. Physiol., 33:227-232, 2004.

[22] B. Rouzaire-Dubois, G. Ouanounou, S. O'Reagan, and J-M. Dubois. Sodium-dependent activity of aquaporin-1 in rat glioma cells: a new mechanism of cell volume regulation. Eur. J. Physiol., 457:1187-1198, 2009.

[23] G. Sjøgaard, R.P. Adams, and B. Saltin. Water and ion shifts in skeletal muscle of humans with intense dynamic knee extension. Am J Physiol., 248:190-196, 1985. 
Centre de recherche INRIA Bordeaux - Sud Ouest

Domaine Universitaire - 351, cours de la Libération - 33405 Talence Cedex (France)

Centre de recherche INRIA Grenoble - Rhône-Alpes : 655, avenue de l'Europe - 38334 Montbonnot Saint-Ismier

Centre de recherche INRIA Lille - Nord Europe : Parc Scientifique de la Haute Borne - 40, avenue Halley - 59650 Villeneuve d'Ascq

Centre de recherche INRIA Nancy - Grand Est : LORIA, Technopôle de Nancy-Brabois - Campus scientifique 615, rue du Jardin Botanique - BP 101 - 54602 Villers-lès-Nancy Cedex

Centre de recherche INRIA Paris - Rocquencourt : Domaine de Voluceau - Rocquencourt - BP 105 - 78153 Le Chesnay Cedex

Centre de recherche INRIA Rennes - Bretagne Atlantique : IRISA, Campus universitaire de Beaulieu - 35042 Rennes Cedex

Centre de recherche INRIA Saclay - Île-de-France : Parc Orsay Université - ZAC des Vignes : 4, rue Jacques Monod - 91893 Orsay Cedex

Centre de recherche INRIA Sophia Antipolis - Méditerranée : 2004, route des Lucioles - BP 93 - 06902 Sophia Antipolis Cedex 